\title{
Assessment of Blood Pressure Control among Hypertensive Patients in Zewditu Memorial Hospital, Addis Ababa, Ethiopia: A Cross-Sectional Study
}

\author{
Degarege Yazie ${ }^{1}$, Workineh Shibeshi², Minyahil Alebachew ${ }^{2}$ and Alemseged Beyene Berha ${ }^{2^{*}}$ \\ ${ }^{1}$ Department of Pharmacy, Menelik II Referal Hospital, Addis Ababa, Ethiopia \\ ${ }^{2}$ Department of Pharmacology and Clinical Pharmacy, School of Pharmacy, College of Health Sciences, Addis Ababa University, Addis Ababa, Ethiopia
}

\begin{abstract}
Background: Hypertension is a major global concern and public health problem affecting more than one billion individuals worldwide. It is risk factor for cardiovascular diseases (CVDs). The main objective of this study was to determine the prescription pattern of antihypertensive medications among patients on follow-up at cardiac clinic of Zewditu Memorial Hospital.
\end{abstract}

Method: A cross-sectional retrospective study design was used to collect the data from the patient chart by reviewing the hypertension patient's medical record.

Result: Out of the 356 patient cards reviewed, $204(57.3 \%)$ were for females and with the mean age of 56.2 \pm 12.4 years. Among total study participants, $124(34.8 \%)$ had co-morbid conditions. Majority of the patients were on combination therapy 252 (70.8\%). Angiotensin converting enzyme inhibitor (ACEI), 215 (60.4\%) was the most prescribed drug. Blood pressure was controlled in 107 (30.1\%) of patients. About 140 (39.3\%) and 134 (37.6\%) hypertensive patients were appropriately prescribed according to national guideline 2014 and Joint National Committee (JNC 8) guideline, respectively. The result of the multivariate analysis showed that there was a positive statistically significant association between inappropriate prescription pattern of antihypertensive medications with age $\geq 60$ years $(P=0.01)$ and uncontrolled blood pressure $(B P)(P=0.001)$.

Conclusion: In conclusion, the general use of antihypertensive medications seemed not to be in accordance with the Ethiopia national guideline.

Keywords: Blood pressure control; Hypertensive patients; Antihypertensive medication

Abbreviations: ACEI: Angiotensin Converting Enzyme Inhibitor; ARB: Angiotensin II Receptor Antagonist; BB: Beta-blockers; BP: Blood Pressure; CCB: Calcium Channel Blocker; CHD: Coronary Heart Disease; CHF: Congestive Heart Failure; CKD: Chronic Kidney Disease; DBP: Diastolic Blood Pressure; DM: Diabetes Mellitus; JNC: Joint National Committee; LVH: Left Ventricular Hypertrophy; SBP: Systolic Blood Pressure; STG: Standard Treatment Guideline

\section{Background}

Hypertension is a major global concern and public health problem affecting more than one billion individuals worldwide and is one of the key preventable risk factors for cardiovascular events $[1,2]$. The prevalence is projected to increase to $29.0 \%$ in men and $29.5 \%$ in women by 2025 [3]. There are several published guidelines on classification of hypertension which help physicians in reaching to proper control of hypertension [4]. Blood pressure (BP) for adults age $\geq 18$ can be categorized based on stage: pre-hypertension is systolic blood pressure (SBP) $120-139 \mathrm{~mm} \mathrm{Hg}$ or diastolic blood pressure (DBP) $80-89 \mathrm{~mm} \mathrm{Hg}$, stage 1 hypertension SBP 140-159 mm Hg or DBP 90-99 mm Hg and stage 2 hypertension for SBP $\geq 160 \mathrm{~mm} \mathrm{Hg}$ or DBP $\geq 100 \mathrm{mmHg}[5,6]$.

According to American Society of Hypertension and the International Society of Hypertension treatment with drugs should be started in patients with blood pressures $>140 / 90 \mathrm{~mm} \mathrm{Hg}$ in whom nonpharmacology treatments have not been effective (R). In patients with stage 2 hypertension drug treatment should be started immediately after diagnosis, usually with a 2 drug combination [5]. There is a wide range of variation in the type of antihypertensive drug class prescription by physicians in different parts of the world [7]. High BP can be treated medically using several classes of drugs such as angiotensin converting enzyme inhibitors (ACEIs), angiotensin II receptor antagonists (ARBs), beta-blockers (BBs), diuretics and calcium channel blockers (CCBs) being the primary drugs used in treatment $[8,9]$. Many new effective antihypertensive drugs became available, which gives hypertensive patients more opportunities to have their blood pressure controlled with fewer side effects [4].

Hypertension has been shown as a major risk factor not only for the development of diabetes but also for the development of microvascular complications like nephropathy, retinopathy and macrovascular complications like coronary artery disease, stroke, peripheral vascular disease (PVD) in diabetic patients [10]. Hypertension and diabetes mellitus (DM) frequently coexist which increases with age [11] The choice of anti-hypertensive drug class is influenced by many factors such as age, race, stage of hypertension and the presence of concomitant disease $[12,13]$. The Joint National Committee (JNC) seventh report recommended that the $\mathrm{BP}$ in diabetics should be controlled to levels of

*Corresponding author: Alemseged Beyene Berha, Clinical Pharmacy Unit and Experiential Training, Department of Pharmacology and Clinical Pharmacy, Schoo of Pharmacy, College of Health Sciences, Addis Ababa University, Addis Ababa, Ethiopia, Tel: +251913258012; E-mail: alembeyene98@gmail.com

Received May 26, 2018; Accepted June 22, 2018; Published June 30, 2018

Citation: Yazie D, Shibeshi W, Alebachew M, Berha AB (2018) Assessment of Blood Pressure Control among Hypertensive Patients in Zewditu Memorial Hospital, Addis Ababa, Ethiopia: A Cross-Sectional Study. J Bioanal Biomed 10: 80-87. doi:10.4172/1948-593X.1000210

Copyright: @2018 Yazie D, et al. This is an open-access article distributed unde the terms of the Creative Commons Attribution License, which permits unrestricted use, distribution, and reproduction in any medium, provided the original author and source are credited. 
130/80 mmHg or lower [14]. In adults without compelling indications initial therapy should include thiazide diuretics, ACEIs, CCBs, ARBs or BBs (in those younger than 60 years of age) [7]. But in the presence of high risk conditions, therapy should be started with ACE inhibitors, ARB's, beta blockers or calcium channel blockers alone or in combination with thiazides [14]

Several guidelines for hypertension management recommended rational combination of drugs of different mechanisms of action for effective control of BP because mono-therapy will not achieve the optimal BP goal in the majority of patients [13,15-17], particularly in patient with severe hypertension $[17,18]$. Compliance will also increase due to fewer side effects $[13,16]$. Low dose combinations can doubly benefit the patients by being more efficacious and more safe thereby has the potential to improve the quality of life of the patient [19]. Most guidelines recommended that thiazide type diuretics or CCBs or ACEIs could be the first-choice for initial therapy and BBs are not a preferred initial therapy for hypertension [8]. The benefits of ACEIs in preventing cardiovascular events in patients with diabetes mellitus, heart failure, hypertensive renal insufficiency and diabetic nephropathy have been documented [20]. Patients with high blood pressure often have no clinical symptoms until organ damage begins, giving the disease its reputation as a "silent killer" [21].

More than $80 \%$ of deaths from hypertension and associated cardiovascular diseases occur in low and middle income countries and this is particularly common among people of low socio-economic status [22]. As in any other developing countries, Ethiopia is challenged by the growing magnitude of chronic non-communicable diseases (NCDs) which created a double burden on the population and the health system which is already hard hit by communicable diseases. Available population based studies indicated high prevalence of NCDs including hypertension, other cardiovascular diseases, diabetes etc, in both rural and urban settings [23]. On the other hand, the Ethiopian health system is designed primarily for the prevention and control of communicable diseases, giving very little emphasis on NCDs [24].

The status of hypertension management is far from being satisfactory [25]. Many studies have demonstrated that lifestyle modifications and adherence to appropriate drug treatments are sufficient to maintain blood pressure at optimal levels. However, available evidence has also shown that about $75 \%$ of hypertensive patients do not have optimal blood pressure control [26]. Despite of the availability of a wide range of medications, and increasing awareness of the dramatic impact of BP treatment on reduction of cardiovascular morbidity and mortality, hypertension is still uncontrolled [27]. Control may remain poor as a result of limited resources, poor adherence to medication as well as inappropriate choice of medication. Published evidence has shown that only $50 \%$ of physicians complied with guideline recommendations [28]. Therefore, the aim of this study was to assess the management of hypertension among hypertensive patients in Zewditu Memorial Hospital.

\section{Methods}

\section{Study setting and period}

The study was conducted at Zewditu Memorial Hospital located in centre of Addis Ababa, Ethiopia. It is a teaching and general referral hospital affiliated with Addis Ababa University and under the administration of Addis Ababa city health bureau. This hospital was selected because it is a teaching and referral hospital that provides organized hypertension follow-up care. There were around 274 hypertension patients on treatment receiving services per month in ambulatory cardiac clinic. Around 112,172 patients were served per year in the hospital. The hospital has 872 clinical and non-clinical staff members. The services given in the hospital include hospital pharmacy, cardiac clinic, diabetic clinic, internal medicine, surgery, paediatrics, genecology/obstetrics, psychiatry, neurology, dermatology, HIV clinic, dental clinic and dialysis centre. The study was conducted during the period from July 24-August 23, 2016.

\section{Study design}

A retrospective cross-sectional study was employed to conduct the study. Simple random sampling technique was used in the data collection. Data was collected from the patient chart by reviewing the patient's medical record of hypertension patients.

\section{Sample size and sampling method}

Sample size was calculated by assuming 0.3 of proportions (p) of hypertension since the prevalence of hypertension in Addis Ababa is estimated to $30 \%$ of total population [28], 5\% marginal error (d) and confidence interval of $95 \%$. Based on this assumption, the sample size was calculated by single population proportion formula $n=((\mathrm{Za} / 2) 2 \times \mathrm{p}$ $(1-p)) / d 2$.This yield sample size of 323 . Since the source population was less than 10,000 the sample size was adjusted with correction formula and with the assumption of $10 \%$ incomplete data, the final sample size was 356 hypertension patients' card.

\section{Data collection and management}

Four nurses were recruited and trained for one day to give standardized instruction and clarify data abstraction format. One nurse was assigned to supervise the data collection process. The supervisor and principal investigator closely followed the data collection process at the spot. Data was collected by using a data abstraction format. Although supervision was carried out, daily checking of the collected data was made during data collection by principal investigator. Data collected include sex, age, systolic and diastolic $\mathrm{BP}$ readings, duration of antihypertensive therapy, and stage of hypertension, co-morbid conditions (diabetes mellitus, cardiovascular disease and chronic kidney disease), nature of therapy and the antihypertensive medications prescribed.

\section{Data quality assurance}

The completeness of the data collected from the survey was checked by the principal investigator in order to maintain consistency. The data abstraction format for the study was prepared in the English language. The data collectors as well as the supervisor were given one day training on the overall data collection procedure. Five percent of the sample was pre-tested to check acceptability and consistency two weeks before the actual data collection.

\section{Data analysis}

The data was entered in to computer using EPI-info 3.5.4 software. Data checking and cleaning was done by principal investigator on daily basis during collection before actual analysis. Analysis was done using statistical software for social sciences (SPSS) version 20. Descriptive data was generated and placed in terms of frequency and percentage. Results were expressed as proportions and as Means \pm Standard Deviations (SD). The level of BP control was assessed by using the average of three BP records obtained from three different visits [29]. Univariate and multivariate logistic regression was used to analyze the associations between dependent variable and independent variables by 
Citation: Yazie D, Shibeshi W, Alebachew M, Berha AB (2018) Assessment of Blood Pressure Control among Hypertensive Patients in Zewditu Memorial Hospital, Addis Ababa, Ethiopia: A Cross-Sectional Study. J Bioanal Biomed 10: 80-87. doi:10.4172/1948-593X.1000210

using crude odds ratio (COR) and adjusted odds ratio (AOR) at $95 \%$ confidence level. A p-value of less than 0.05 was considered statistically significant.

\section{Ethical consideration}

Ethical clearance was obtained from Ethical Review Board of School of Pharmacy, Addis Ababa University. The aim and objectives of the study was explained to the health facility before data collection and permission was obtained from the hospital medical director to access patients' medical record and conduct the study. Consent was not obtained as this study was based on retrospective review of patient medical charts. Privacy and confidentiality was guaranteed by storing data in a safe place, by excluding identifiers (patients' files were coded and name was not recorded).

\section{Results}

\section{Socio-demographic and clinical characteristics}

A total of 356 hypertensive patients on treatment were included. Out of the 356 patients studied, majority 204 (57.3\%) were females. The mean age of patients was $56.2 \pm 12.4$ years (range 23 to 92 years) .The mean duration on antihypertensive therapy was $11.27 \pm 8.34$ years (range of 1-51years). Among hypertensive patients 151 (42.4\%) have been taking antihypertensive therapy for more than 10 years while 114 (32\%) have been taking antihypertensive drugs for 5 to10 years and 91 (25.6\%) have been taking antihypertensive drugs for less than 5 years. Age groups were divided into different categories namely, 20-29 years 8 (2.2\%), 30-39 years 22 (6.2\%), 40-49years 70 (19.7\%), 50-59 years 118 (33.1\%) and $\geq 60$ years 138 (38.8\%). Out of the total study participants, $124(34.8 \%)$ hypertensive patients had co-morbid conditions. Among the co-morbid conditions 78 (21.9\%) patients were diabetic, $17(4.8 \%)$ patients had CKD, $8(2.2 \%)$ had CHF, 4 (1.1\%) had CHD and $1(0.3 \%)$ had LVH, 10 (2.8\%) had DM and CKD (Table1).

\section{Appropriateness of the prescription pattern and frequency of antihypertensive medications}

Out of the total study subjects, 140 (39.3\%) hypertensive patients appropriately prescribed antihypertensive medications according to Ethiopian STG 2014 and 134 (37.6\%) hypertensive patients appropriately used according to The Eighth Joint National Committee (JNC 8) guideline (Figure 1).

The overall utilization pattern of antihypertensive agents showed that ACEI was the most frequently prescribed class of drugs 215 (60.4\%) followed by CCB 211 (59.3\%), BB 167 (46.9\%), diuretic 114 (32.0\%), Central alpha-2 agonist 9 (2.5\%) and ARB 8 (2.3\%) (Table 2).

\section{Antihypertensive therapy}

Among the study participants, 104 (29.2\%) were on monotherapy and $252(70.8 \%)$ were on combination therapy. Among the study participants who received mono-therapy the most prescribed drugs were ACEI 46 (12.9\%), CCB 38 (10.67\%), diuretic 9 (2.5\%), BB 4 (1.1\%), CA2 4 (1.1\%) and ARB 3 (0.84\%).Concerning patients who were on combination antihypertensive drugs therapy 149 (41.9\%) of patients were on two antihypertensive drugs. The most frequently used combinations were ACEI+CCB 35 (9.8\%), $\mathrm{CCB}+\mathrm{BB} 33$ (9.3\%), ACEI + BB 31 (8.7\%) of the total patients (Table 3). Ninety two (25.8\%) patients were on three drugs and among these 46 (12.9\%) were on $\mathrm{ACEI}+\mathrm{CCB}+\mathrm{BB}$ and the rest of the triple therapy combinations are shown in Table 4. Eleven $(3.1 \%)$ patients were received four antihypertensive drugs and among these the most prescribed combinations were diuretic

\begin{tabular}{|c|c|c|}
\hline Variables & Mean \pm SD & Frequency (\%) \\
\hline \multicolumn{3}{|c|}{ Sex } \\
\hline Male & & $152(42.7)$ \\
\hline Female & & $204(57.3)$ \\
\hline Age categories & $56.2 \pm 12.4$ & \\
\hline $20-29$ & & $8(2.2)$ \\
\hline $30-39$ & & $22(6.2)$ \\
\hline $40-49$ & & $70(19.7)$ \\
\hline $50-59$ & & $118(33.1)$ \\
\hline$\geq 60$ & & $138(38.8)$ \\
\hline Duration of therapy (year) & $11.27 \pm 8.34$ & \\
\hline$<5$ & & $91(25.6)$ \\
\hline $5-10$ & & $114(32.0)$ \\
\hline$>10$ & & $151(42.4)$ \\
\hline \multicolumn{3}{|c|}{ Co-morbidity } \\
\hline DM & & $78(21.9)$ \\
\hline CKD & & $17(4.8)$ \\
\hline $\mathrm{CHF}$ & & $8(2.2)$ \\
\hline $\mathrm{CHD}$ & & $4(1.1)$ \\
\hline LVH & & $1(0.3)$ \\
\hline $\mathrm{DM}$ and $\mathrm{CKD}$ & & $10(2.8)$ \\
\hline $\mathrm{DM}$ and $\mathrm{CHF}$ & & $3(0.8)$ \\
\hline $\mathrm{DM}$ and $\mathrm{CHD}$ & & $1(0.3)$ \\
\hline DM and LVH & & $1(0.3)$ \\
\hline $\mathrm{DM}, \mathrm{CKD}$ and $\mathrm{CHF}$ & & $1(0.3)$ \\
\hline \multicolumn{3}{|c|}{$\begin{array}{c}\text { HTN=Hypertension; DM=Diabetes Mellitus; CKD=Chronic Kidney Diseases; } \\
\text { CHF=Congestive Heart Failure; } \\
\text { CHD=Coronary Heart Disease; LVH=Left Ventricular Hypertrophy }\end{array}$} \\
\hline
\end{tabular}

Table 1: The demographic and clinical characteristics of ambulatory hypertensive patients attending Zewditu Memorial Hospital, Ethiopia, 2016.

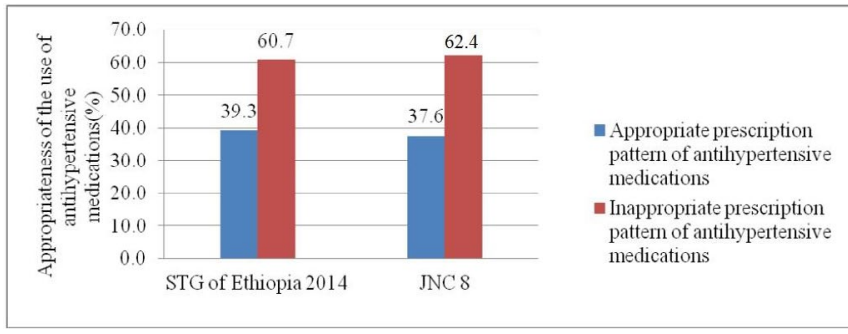

Figure 1: Appropriateness of the prescription pattern of antihypertensive medications according to Ethiopian STG 2014 and JNC 8 guideline among hypertensive patients attending Zewditu Memorial Hospital, Ethiopia, 2016.

\section{$+\mathrm{ACEI}+\mathrm{BB}+\mathrm{CCB}, 10$ (2.8\%) (Table 3).}

\section{Prescription pattern of antihypertensive medications in patients with co-morbidities}

As shown in Table 4, among the hypertensive cases with diabetes mellitus the most prescribed class of drug was ACEI 64 (68.1\%) followed by CCB 54 (57.45\%), BB 34 (36.2\%), diuretic 20 (21.3\%) and ARB and central alpha-2 agonist $1(1.1 \%)$ each. In hypertension patients with CKD, the most prescribed class of drug was CCB 17 (60.7\%) followed by ACEI 16 (57.1\%), diuretic $16(57.1 \%)$ and BB 7 (25.0\%). The most prescribed class of drugs in $\mathrm{CHF}$ hypertension patients was diuretic 9 (75.0\%) followed by ACEI 6 (50.0\%), BB 5 (41.7\%), CCB 4 (33.3\%) and ARB and central alpha-2 agonist $1(8.3 \%)$ each. The most prescribed class of drug in CHD was ACEI 5 (100.0\%) followed by CCB $4(80.0 \%)$, BB $3(60.0 \%)$ and diuretic $1(20.0 \%)$. The commonest prescribed class of drug in LVH hypertension patients was CCB 2 (100.0\%) followed 
Citation: Yazie D, Shibeshi W, Alebachew M, Berha AB (2018) Assessment of Blood Pressure Control among Hypertensive Patients in Zewditu Memorial Hospital, Addis Ababa, Ethiopia: A Cross-Sectional Study. J Bioanal Biomed 10: 80-87. doi:10.4172/1948-593X.1000210

\begin{tabular}{|c|c|}
\hline Antihypertensive group & Frequency (\%) \\
\hline ACEI & $215(60.4)$ \\
\hline Enalapril & $215(60.4)$ \\
\hline CCB & $211(59.3)$ \\
\hline Nifedipine & $195(54.8)$ \\
\hline Amlodipine & $16(4.5)$ \\
\hline BB & $167(46.9)$ \\
\hline Atenolol & $166(46.6)$ \\
\hline Metoprolol & $1(0.3)$ \\
\hline Diuretic & $114(32.0)$ \\
\hline Hydrochlorothiazide & $82(23.0)$ \\
\hline Furosemide & $32(9.0)$ \\
\hline ARB & $8(2.3)$ \\
\hline Losartan & $7(2.0)$ \\
\hline Candesartan & $1(0.3)$ \\
\hline Central alpha-2 agonist & $9(2.5)$ \\
\hline Methyldopa & $9(2.5)$ \\
\hline
\end{tabular}

$\mathrm{ACEI}=$ Angiotensin Converting Enzyme Inhibitor; $\mathrm{BB}=$ Beta Blocker; $\mathrm{ARB}=$ Angiotensin Receptor Blocker $\mathrm{CCB}=$ Calcium Channel Blocker

Table 2: Frequency of administration of individual antihypertensive medications among hypertensive patients attending Zewditu Memorial Hospital, Ethiopia, 2016

\begin{tabular}{|c|c|}
\hline Nature of therapy & Frequency (\%) \\
\hline Mono-therapy & $104(29.2)$ \\
\hline ACEI & $46(12.9)$ \\
\hline CCB & $38(10.7)$ \\
\hline Diuretic & $9(2.5)$ \\
\hline LD & $5(1.4)$ \\
\hline TD & $4(1.1)$ \\
\hline BB & $4(1.1)$ \\
\hline Ca2 & $4(1.1)$ \\
\hline ARB & $3(0.56)$ \\
\hline Dual therapy & $149(41.9)$ \\
\hline ACEI + CCB & $35(9.8)$ \\
\hline ACEI+BB & $31(8.7)$ \\
\hline CCB+BB & $33(9.3)$ \\
\hline ACEI+diuretic & $23(6.5)$ \\
\hline CCB+diuretic & $15(4.2)$ \\
\hline BB+diuretic & $8(2.2)$ \\
\hline diuretics+CA2 & $2(0.84)$ \\
\hline ARB+diuretic & $1(0.3)$ \\
\hline ARB+CCB & $1(0.3)$ \\
\hline Triple therapy & $92(25.8)$ \\
\hline ACEI+BB+CCB & $46(12.9)$ \\
\hline BB+CCB+diuretic & $20(5.6)$ \\
\hline ACEI+BB+diuretic & $12(3.4)$ \\
\hline ACEI+CCB+diuretic & $9(2.5)$ \\
\hline ACEI+diuretic+CA2 & $3(0.8)$ \\
\hline ARB+BB+CCB & $1(0.3)$ \\
\hline ARB+BB+diuretic & $1(0.3)$ \\
\hline Four drugs therapy & $11(3.1)$ \\
\hline ARB+BB+CCB+diuretic $B+C C B+d i u r e t i c$ & $10(2.8)$ \\
\hline Cons & $1(0.3)$ \\
\hline
\end{tabular}

$\mathrm{ACE}=$ Angiotensin Converting Enzyme Inhibitor; $\mathrm{BB}=$ Beta Blocker ARB=Angiotensin Receptor Blocker; $\mathrm{CCB}=$ Calcium Channel Blocker; $\mathrm{CA} 2=$ Central Alpha-2 Agonist; LD = Loop Diuretic; TD =Thiazide Diuretic

Table 3: Antihypertensive therapy among hypertensive patients attending Zewditu Memorial Hospital, Ethiopia, 2016.

\begin{tabular}{|c|c|c|c|c|c|c|c|}
\hline $\begin{array}{c}\text { Co- } \\
\text { morbid } \\
\text { condition }\end{array}$ & N & $\begin{array}{c}\text { ACEI } \\
\mathbf{N}(\%)\end{array}$ & $\begin{array}{c}\text { CCB } \\
\mathbf{N}(\%)\end{array}$ & $\begin{array}{c}\text { BB } \\
\mathbf{N}(\%)\end{array}$ & $\begin{array}{c}\text { Diuretic } \\
\mathbf{N}(\%)\end{array}$ & $\begin{array}{c}\text { ARB } \\
\mathbf{N}(\%)\end{array}$ & $\begin{array}{c}\text { CA2 } \\
\mathbf{N}(\%)\end{array}$ \\
\hline $\mathrm{DM}$ & 94 & $64(68.1)$ & $54(57.45)$ & $34(36.2)$ & $20(21.3)$ & $1(1.1)$ & $1(1.1)$ \\
\hline $\mathrm{CKD}$ & 28 & $16(57.1)$ & $17(60.7)$ & $7(25.0)$ & $16(57.1)$ & 0 & 0 \\
\hline CHF & 12 & $6(50.0)$ & $4(33.3)$ & $5(41.7)$ & $9(75.0)$ & $1(8.3)$ & $1(8.3)$ \\
\hline CHD & 5 & $5(100.0)$ & $4(80.0)$ & $3(60.0)$ & $1(20.0)$ & 0 & 0 \\
\hline LVH & 2 & $1(50.0)$ & $2(100)$ & 0 & 0 & 0 & 0 \\
\hline
\end{tabular}

$\mathrm{DM}=$ Diabetes Mellitus; $\mathrm{CKD}=$ Chronic Kidney Diseases; $\mathrm{CHF}=$ Congestive Heart Failure; $\mathrm{CHD}=$ Coronary Heart Disease; $\mathrm{LVH}=\mathrm{Left}$ ventricular hypertrophy; $\mathrm{ACE}=$ Angiotensin Converting Enzyme Inhibitor; $\mathrm{BB}=$ Beta Blocker $\mathrm{ARB}=$ Angiotensin Receptor Blocker; $\mathrm{CCB}=$ Calcium Channel Blocker; $\mathrm{CA} 2=$ Central Alpha-2 Agonist; N=Frequency

Table 4: Prescription pattern of antihypertensive medications among hypertensive patients with various co-morbidities attending Zewditu Memorial Hospital, Ethiopia, 2016.

by ACEI 1 (50.0\%). But in case of hypertension patients without compelling conditions the most prescribed class of drug was CCB 141 (60.8\%) followed by ACEI 131 (56.5\%), BB 126 (54.3\%), diuretic 76 (32.8\%), CA2 7 (3.0\%) and ARB 6 (2.6\%).

\section{Blood pressure control}

Among the study participants 137 (38.5\%) had a controlled SBP; while 174 (48.9\%) had a controlled DBP. The overall control of BP was achieved in 107 (30.1\%) of the study participants according to national guideline (STG, 2014) (Figure 2).

\section{Determinants of the appropriate prescription pattern of antihypertensive medications}

The association of independent variables with the dependent variable was investigated using both univariate and multivariate logistic regression. Only two variables were shown to have significant association with the appropriateness of the prescription pattern of antihypertensive medications. The result of the multivariate analysis showed that there was a positive statistical significant association between inappropriate prescription pattern of antihypertensive medications and age $\geq 60$ years $(\mathrm{P}<0.05)$ and uncontrolled $\mathrm{BP}(\mathrm{P}<0.05)$. Patients with controlled $\mathrm{BP}$ were two fold more likely to have appropriate prescription pattern of antihypertensive medications when compared patients with uncontrolled BP (AOR=0.45, 95\% CI: 0.27-0.73, $\mathrm{P}=0.001)$ (Table 5).

\section{Discussion}

This study was a retrospective cross sectional study designed to assess the pattern of antihypertensive medications in order to obtain an insight into the current utilization of antihypertensive drugs in Zewditu Memorial Hospital. Blood control status was found to be associated with inappropriate prescription pattern of antihypertensive medications when compared patients with controlled BP. The result of the current study showed that only one third of hypertensive patients

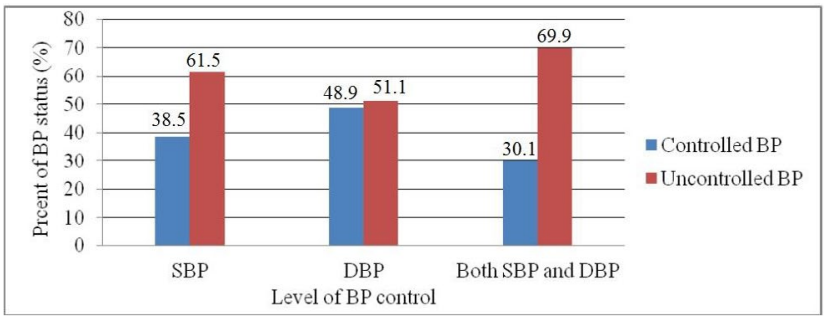

Figure 2: Blood pressure control among hypertensive patients attending Zewditu Memorial Hospital, Ethiopia, 2016. 
Citation: Yazie D, Shibeshi W, Alebachew M, Berha AB (2018) Assessment of Blood Pressure Control among Hypertensive Patients in Zewditu Memorial Hospital, Addis Ababa, Ethiopia: A Cross-Sectional Study. J Bioanal Biomed 10: 80-87. doi:10.4172/1948-593X.1000210

\begin{tabular}{|c|c|c|c|c|c|}
\hline \multirow[t]{2}{*}{ Variables } & \multicolumn{2}{|c|}{$\begin{array}{l}\text { Appropriate prescription pattern of antihypertensive } \\
\text { medications }\end{array}$} & \multirow[t]{2}{*}{$\operatorname{COR}(95 \% \mathrm{Cl})$} & \multirow[t]{2}{*}{$\operatorname{AOR}(95 \% \mathrm{Cl})$} & \multirow[t]{2}{*}{ P-value } \\
\hline & Yes (\%) & No $(\%)$ & & & \\
\hline \multicolumn{6}{|c|}{ Sex } \\
\hline Female & $79(22.2)$ & $125(35.1)$ & 1.00 & 1.00 & \\
\hline Male & $61(17.1)$ & $91(25.6)$ & $0.94(0.62,1.45)$ & $1.14(0.71,1.82)$ & 0.58 \\
\hline \multicolumn{6}{|c|}{ Age category } \\
\hline$<60$ years & $72(20.2)$ & $146(41.0)$ & 1.00 & 1.00 & \\
\hline$\geq 60$ years & $68(19.1)$ & $70(19.7)$ & $0.51(0.33,0.79)$ & $0.54(0.34,0.87)$ & 0.01 \\
\hline \multicolumn{6}{|c|}{ Co-morbid condition } \\
\hline HTN alone & $92(25.84)$ & $140(39.33)$ & 1.00 & 1.00 & \\
\hline DM & $36(10.1)$ & $42(11.8)$ & $0.77(.46,1.29)$ & $0.83(0.48,1.43)$ & 0.50 \\
\hline CKD & $7(1.97)$ & $10(2.8)$ & $0.94(.35,2.56)$ & $0.89(0.32,2.50)$ & 0.82 \\
\hline $\mathrm{DM}$ and $\mathrm{CKD}$ & $4(1.12)$ & $6(1.69)$ & $0.99(.27,3.59)$ & $0.90(0.24,3.36)$ & 0.88 \\
\hline $\mathrm{CHD}$ & $1(0.3)$ & $4(1.1)$ & $2.63(0.39,23.90)$ & $3.14(0.33,30.02)$ & 0.32 \\
\hline \multicolumn{6}{|c|}{ BP status } \\
\hline Controlled & $57(16.0)$ & $50(14.0)$ & 1.00 & 1.00 & \\
\hline Uncontrolled & $83(23.3)$ & $166(46.7)$ & $2.28(1.44,3.62)$ & $0.45(0.27,0.73)$ & 0.001 \\
\hline
\end{tabular}

Others*: $B B, A R B, A R B+D i u r e t i c, C A, A R B+C C B, C C B+B B, C A+$ Diuretic, $C C B+B B+D i u r e t i c$,

$A R B+C C B+B B+D i u r e t i c, A R B+B B+C C B, A R B+B B+$ Diuretic and $A C E I+D i u r e t i c+C A_{2}$.

$\mathrm{ACEI}=$ Angiotensin Converting Enzyme Inhibitor; BB=Beta Blocker; ARB=Angiotensin Receptor Blocker; CCB=Calcium Channel Blocker; CA2=Central Alpha-2 Agonist; $\mathrm{HTN}=$ Hypertension; DM=Diabetes Mellitus CKD=Chronic Kidney Diseases; CHD=Coronary Heart Disease

Table 5: Determinants of the appropriate use of antihypertensive medications among hypertensive patients attending Zewditu Memorial Hospital, Ethiopia, 2016.

on pharmacologic treatment had a controlled BP 107 (30.1\%). The level of BP control found in this study was similar to the study conducted in Nigeria (30.5\%) [20]. The present study revealed similar result with the health centre based study (31\%) [29]. This similarity might be due to the study conducted in the same geographic area and similar inclusion criteria. But the level of BP control found in this study was lower than the studies from Saudi Arabia (72\%) [30], Italy (43.23\%) [31] and Togo (54.4\%) [32]. This difference in the level of BP control might be due to a more aggressive therapy with two or more drug combinations. The racial difference might have contributed to this inconsistency. Moreover, the level of BP control in the present study a little bit higher than the study performed two years ago (26.2\%) by Adugna, this difference might be due to the use of different guidelines as a standard. But the level of BP control in the present study was higher than the study performed (15\%) by Abera et al. [33].This difference might be due to patients with comorbid conditions difficult to achieve target BP easily and in case of the study conducted by Abera et al. all hypertension patients had DM.

Some of the reasons attributed to the inadequate BP control were reported to be poor compliance with medications which is strongly determined by poverty, excessive salt intake, use of non-steroidal antiinflammatory drugs (NSAIDs) and inappropriate combinations with inadequate dosing of antihypertensive agents was revealed by Etuk et al. [20]. The result of the current study showed that only 140 (39.3\%) and $133(37.4 \%)$ of hypertensive patients on pharmacologic treatment had appropriate use of antihypertensive medications according to Ethiopian national guideline (STG 2014) and JNC 8 guideline respectively. This small difference might be because of the prescription pattern of antihypertensive medications in JNC 8 consider the racial difference. Among the limitation of this study, the status of patients' compliance to prescription and patients' lifestyle change were significantly affected the outcome of medication but were not reported in this study. The prevalence of appropriate use of antihypertensive medications found in this study (39.3\%) was lower than the studies from Saudi Arabia
(60\%) [30], Malaysia (85.3\%) [34], Ethiopia (97.0\%) [1] and published evidence has shown that $50 \%$ of physicians complied with guideline recommendations $[28,35]$. In the present study appropriate use of antihypertensive medications was found to be low which might be associated with the adherence of the prescriber to the national STG.

The results of the present study indicated that the proportion of females with hypertension $204(57.3 \%)$ was high. This study was in line with two Ethiopian studies [1,36] and Nigeria study [16]. In contrary, the present study was inconsistence with other studies which were conducted in Bangladesh [37] and India [38]. The result of the multivariate analysis showed that age was significantly associated with inappropriate prescription pattern of antihypertensive medications in patients age $\geq 60$ years ( $\mathrm{AOR}=0.54,95 \% \mathrm{CI}: 0.34,0.87, \mathrm{P}=0.01$ ). This study also revealed that $256(71.9 \%)$ of hypertension patients were age over 50 years. The current study was in line with the study conducted in Ethiopia [1] and India [39]. These results showed that age may affect the incidence of hypertension.

The classes of antihypertensive agents prescribed in this study were ACEI 215 (60.4\%), CCB 211 (59.3\%), BB 167 (46.9\%), diuretic 114 (32.0\%), CA2 9 (2.5\%) and ARB 8 (2.3\%). Results resembling such pattern in the use of antihypertensive drugs were observed in a tertiary care hospital based study from India which reported that the most frequent group of antihypertensive medication was ACEI (44.25\%) by Arief et al. [40] and the study conducted in the current study area two years ago [41]. ACEI was the first line antihypertensive drug for hypertension patients and the result of the study was comparable with the current national guidelines of Ethiopia 2014 (STG).

In the present study, the overall prescription pattern of antihypertensive medication was inconsistent with a study conducted in a general hospital in China, CCB (58.0\%), ARB (34.2\%), ACEI (17.5\%), BB (16.9\%) and diuretic (16.7\%) [8]. This difference might be due to age variation of the study participants. In the present study the majority 
of the patients were younger than 60 years with a mean age of $56.2 \pm$ 12.4 but the study conducted in China the majority of the patients were older than 60 years with the mean age was $61.3 \pm 13.7$ years.

According to the study conducted in India by Cidda et al. [9], the most common drug class involved in the study was ARB (42.24\%). This discrepancy might be due to cost variation across the study places. The result of cost evaluation showed that diuretics had the lowest cost and ARBs had the highest cost per day [34,42].The study also conducted in Malaysia the most prescribed antihypertensive medications were BBs (85.9\%) [34]. The discrepancy of the use of BB over ACEI might be associated with the prevalence of types of co-morbid condition since the majority of the patients in Abdulameer et al. [28] study occurred with CHD. On the contrary, the study conducted in Addis Ababa health centres showed that the most commonly used antihypertensive drugs were thiazide diuretics (36.5\%). This difference in frequent use of ACEI over diuretic might be due to the high prevalence of co-morbid conditions (i.e. DM and CKD).

In the current study, BB which was considered as a second line antihypertensive agent; it was reported to be used in 167 (46.9\%) of the hypertensive patients. This discrepancy might have resulted from lack of adherence to national guideline (STG). Since BB is a second line drug this result showed that BB was inappropriately prescribed. Beta blockers should only be prescribed when compelling conditions such as CHF and CHD are co-diagnosed regards to Ethiopian STG 2014. But in case of this study these two diseases were 17 (4.8\%) only.

The result of the study showed that majority of the patients were on combination therapy $252(70.8 \%)$ which was consistent with the study conducted in Nigeria, 2008 (80\%) [20] in Togo, 2012 (81.40\%) [32] and the study conducted in Ethiopia by Adugna, (83.1\%) [41]. The present result and the study conducted by Adugna [41] showed that combination therapy practice had consistency. But higher than the study which was done in Ethiopia (34.5\%) [1], Bangladesh (38.4\%) [37] and China $(37.1 \%)$ [8]. The higher prescription rate of combination therapy might be due to the longer duration of hypertension therapy and being high risk patients. Moreover, according to the study conducted in Addis Ababa health centres, Ethiopia the majority of the patients were on mono-therapy (78.9\%) [29]. The present study was inconsistent with the above study might be due to the hospital based patients presented with severe and more complicated hypertension since they are referred from the health centre.

Out of 104 (29.2\%) patients who received mono-therapy ACEI, $46(44.2 \%)$ was the most prescribed drug. This study was similar to a study conducted in India which showed that ACEI (38.25\%) was the most prescribed drug [40]. On contrary, the study conducted in Addis Ababa health centres showed that Ca2 (methyldopa) was the most common mono-therapy used (20.8\%) [29]. This discrepancy might be because of the difference in level of knowledge on the management of hypertension.

According to the Ethiopian guideline 2014, mono-therapy was only preferred if the target blood pressure was achieved successfully by that particular antihypertensive agent. But if the target blood pressure was not achieved with one agent, then poly-therapy should be introduced and low doses of two or more agents should be given. Nature of therapy was also found to have association with inappropriate prescription pattern of antihypertensive medications in that when compared with mono-therapy. The most frequently used two drug combinations were ACEI+CCB, 35 (9.8\%).This combination is strongly supported by the national guideline for patients without co-morbid conditions [43] hence the prescribers might be familiar with this combination. This study was similar to a study conducted in India [40]. On the other hand, the study conducted in Nigeria CCB+diuretic (36.6\%) were the most frequently used combinations [44]. This difference might be due to the variations of the prevalence of the types of co-morbid condition and the recommended guidelines. Moreover, the study conducted by Adugna [41] in Zewditu Memorial Hospital, Ethiopia the most frequently used two drug combination was ACEI+BB (14.2\%). This difference might be associated that in the present study the prescriber's awareness for the national guideline was improved.

Regarding patients who were on three antihypertensive therapies $\mathrm{ACEI}+\mathrm{CCB}+\mathrm{BB}$ combinations were the most prescribed drugs. This study showed similarity with the survey conducted in India [18]. With respect to patients receiving four antihypertensive drugs therapy the most frequently used combinations were $\mathrm{ACEI}+$ diuretic+BB+CCB. On the other hand, the study conducted in Nigeria the most frequently used combinations were $\mathrm{ACEI}+\mathrm{CCB}+$ diuretic+methyldopa [16]. The observed difference of this study with the above study might be attributed to the possibility of some patients presenting with multiple co-morbid conditions.

The result of the current study showed that among the study participants on mono-therapy $41(40.6 \%)$ had controlled blood pressure while among patients on combination therapy 68 (26.7\%) had controlled BP. The current study showed similarity with a study conducted in Nigeria with mono-therapy $41.4 \%$ had BP controlled and with combination therapy $17.9 \%$ had controlled BP [17]. In contrary, another study conducted in Nigeria showed that with mono-therapy $34.4 \%$ had BP controlled and with combination therapy $47.2 \%$ had controlled BP [45]. The higher proportion of controlled BP with monotherapy when compared with combination therapy might be associated with the more appropriate prescribed pattern of mono-therapy.

According to the present study the most prevalent co-morbid disease was DM. Similarly, the most prevalent co-morbid disease was DM according to many studies reported $[1,15,36,38,46]$. The increase incidence of DM in hypertensive patients might be due to factors which have been proposed to contribute to hypertension in diabetes: hyperinsulinemia, extracellular fluid volume expansion and increased arterial stiffness [47]. Among the hypertensive cases with DM, the most prescribed class of drugs was ACEI 64 (68.1\%). This study showed similarity with a study conducted in Saudi Arabia; ACEI (about 75.0\%) [30] and in diabetic clinic at Zewditu Memorial Hospital, Ethiopia; ACEI (84\%) [33]. ACE-inhibitors are the only class of drugs that are often prescribed to diabetic hypertensive patients, as these drugs prevent the chance of occurrence of retinopathy and other related complications [40]. On the other hand, the study conducted in Nigeria the most prescribed class of drug was diuretic (61.5\%) [20] and in China CCB (61.7\%) [8]. These differences in result might be due to the status of prescribers' awareness about the national and international guidelines and the presence of other compelling conditions.

The commonest prescribed class of drug in CKD hypertension patients was CCB 17 (60.7\%). This study was in line with the studies conducted in China CCB (56.4\%) [8] and Saudi Arabia CCB (about 40\%) [30]. But the study conducted in Nigeria the first choice drug was diuretic (91.7\%) [20]. In the current study, CCB was preferred to treat hypertensive patients with $\mathrm{CKD}$ in contrast to guideline that recommended ACEI/ARB. Seventh Report of the Joint National Committee guidelines advocate ACEIs as the favored drug for hypertension associated with chronic kidney disease, diabetes or congestive heart failure [35]. The most prescribed class of drug in 
CHF hypertension patients were diuretics 9 (75.0\%). Similar study was conducted in Nigeria and the most prescribed drugs were diuretic (100\%) [20]. On the other hand, the study conducted in China has shown that CCB (61.7\%) was the most prescribed drug [8]. In the current study the preferred drug was diuretic as first line to treat hypertensive patients with CHF in contrast to guideline that recommended ACEI/ARB + metoprolol/carvedilol. The present study showed that the physicians might not be paid sufficient attention to the benefits of ACE inhibitor as the first line drug in treatment of co-morbidities (CHF and CKD) associated with hypertension.

The most prescribed class of drug in CHD was ACEI 5(100.0\%) followed by CCB 4 (80.0\%). In the present study the preferred drug was ACEI (100.0\%) to treat hypertensive patients with CHD and also it was consistent with the guideline [43]. The commonest prescribed class of drugs in hypertension patients with LVH was CCB 2 (100.0\%). The result of the present study showed that $100 \%$ not in accordance with the national guideline (Ethiopian STG 2014). Since the first choice of hypertension patients with LVH was ACEI/ARB and the second line was thiazide diuretics but $\mathrm{CCB}$ even not an alternative choice according to Ethiopian STG 2014. But in case of hypertension patients without comorbid conditions the most prescribed class of drugs was CCB (60.8\%). This study was in line with the study conducted in China which showed that the most prescribed class of drug was CCB (56.4\%) [8]. In the present study CCB was first choice of class of antihypertensive which might be associated with the racial difference.

\section{Conclusion}

Overall utilization pattern of antihypertensive medications indicates that ACEI and CCB were the most frequently prescribed class of drugs in both mono-therapy and combination therapy. There was underutilization of diuretics in this study, in spite of reasonable evidences backing their prescription. Uncontrolled BP and age $\geq$ 60 years were positively associated with inappropriate prescription pattern of antihypertensive medications. Despite the use of different antihypertensive drugs, most of the patients did not achieve the target blood pressure and the general use of antihypertensive medications, as observed in this study, is not to be in accordance with the national guideline of Ethiopia for management of hypertension.

\section{Declarations}

\section{Ethics approval and consent to participate}

The study was approved by Ethical Review Board of School of Pharmacy, Addis Ababa University. However, no consent was needed as the study was based on retrospective chart review.

Authors' contribution DY conceived the idea for the study, designed it, collected data, performed data analysis, and wrote parts of the introduction, methods, results and discussion sections of the research. MA, WS and $\mathrm{ABB}$ participated in the design, collected data, and contributed to the writing of the methods and results sections. All authors are responsible for the drafting, review and final approval of the manuscript.

\section{Acknowledgement}

The study was partly supported by Addis Ababa University. The authors also thank Addis Ababa City health bureau for sponsoring first author and all participants of the study.

\section{References}

1. Shukrala F, Gabriel T (2015) Assessment of prescribing, dispensing, and patient use pattern of antihypertensive drugs for patients attending outpatient department of Hiwot Fana Specialized University Hospital, Harar, Eastern Ethiopia. Drug Des Devel Ther 9: 519-523.

2. James PA, Oparil S, Carter BL, Cushman WC, Dennison-Himmelfarb C, et al. (2014) evidence-based guideline for the management of high blood pressure in adults: report from the panel members appointed to the Eighth Joint National Committee (JNC 8). JAMA 311: 507-520.

3. Kearney PM, Whelton M, Reynolds K, Muntner P, Whelton PK, et al. (2004) Global Burden Of Hypertension: P 512. J Hypertens 22.

4. Al-Drabah E, Irshaid Y, Yasein N, Zmeili S (2013) Prescription pattern of antihypertensive drugs in Family Practice Clinics at Jordan University Hospital. Med Sci 2: 496-488.

5. Weber MA, Schiffrin EL, White WB, Mann S, Lindholm LH, et al. (2014) Clinical practice guidelines for the management of hypertension in the community. $J$ clin hypertens 16: 14-26.

6. Malaysia. $\mathrm{MoH}$ (2013) Clinical practice guidelines: management of hypertension In. Edited by Hypertension. MSo, 4th edn.

7. Sepehri G, Talebizadeh N, Mirzazadeh A, Mohsenbeigi M (2006) The patterns of antihypertensive drug prescription by cardiologists in Kerman province of Iran. Pharmacoepidemiol Drug Saf 17: 180-185

8. Cheng $H$ (2011) Prescribing pattern of antihypertensive drugs in a general hospital in central China. Int J clin pharm 33: 215-220.

9. Cidda M, Mateti UV, Batchu MK, Martha S (2014) Study of prescribing patterns of antihypertensives in South Indian population. Int J Basic Clin Pharmacol 3: 303-307.

10. Khrime D, Kumar A, Pandey AN, Bansal N, Sharma U, et al. (2015) Antihypertensive drug utilization pattern and awareness in diabetic hypertensive patients at tertiary care centre. Int JRes Med Sci 2: 461-465.

11. Kousalya K, Chirumamilla S, Manjunath S, Ramalakshmi S, Saranya P, et al. (2012) Prescribing trend of antihypertensive drugs in hypertensive and diabetic hypertensiv e patients. Asian J Pharm and Clin Res 5: 22-23.

12. Shah J, Khakhkhar T, Bhirud S, Shah RB, Date S (2013) Study of utilization pattern of anti-hypertensive drugs in hypertensive diabetic patients with or without reduced renal function at tertiary care teaching hospital. Int J Med Sci Public Health 2: 175-180.

13. Rimoy G, Justin-Temu M, Nilay C (2008) Prescribing patterns and cost of antihypertensive drugs in private hospitals in Dar es Salaam, Tanzania. East Cent. Afr. J. Pharm. Sci. 11: 69-73.

14. Chobanian AV, Bakris GL, Black HR, Cushman WC, et al. (2003) Seventh report of the joint national committee on prevention, detection, evaluation, and treatment of high blood pressure. Hypertens 42: 1206-1252.

15. Ukwe CV, Ubaka CM (2012) Antihypertensive drug prescribing in a tertiary hospital in Eastern Nigeria. Trop J Pharm Res 11: 297-305.

16. Olanrewaju T, Aderibigbe A, Busari O, Sanya E (2010) Antihypertensive drug utilization and conformity to guidelines in a sub-Saharan African hypertensive population. Inte $\mathrm{J}$ clin pharmacol ther 48: 68-75.

17. Oke O, Adedapo A (2015) Antihypertensive drug utilization and blood pressure control in a Nigerian hypertensive population. Gen Med: Open Access 3: 169.

18. Khurshid F, Aqil M, Alam MS, Kapur P, Pillai KK (2012) Antihypertensive medication prescribing patterns in a university teaching hospital in South Delhi. Int J Pharm Sci Res 3: 2057-2063.

19. Kaur S, Gupta S, Kumar D, Lal M, Gilani Z (2012) Prescribing pattern of antihypertensive drugs in a tertiary care hospital in Jammu: a descriptive study. JK-Pract17: 38-41.

20. Etuk E, Isezuo S, Chika A, Akuche J, Ali M (2008) Prescription pattern of antihypertensive drugs in a tertiary health institution in Nigeria. Ann Afr med 7: 128-132.

21. Karaeren H, Yokuşoğlu M, Uzun Ş, Baysan O, Köz C, et al. (2009) The effect of the content of the knowledge on adherence to medication in hypertensive patients. Anadolu Kardiyol Der 9: 183-188.

22. Kearney PM, Whelton M, Reynolds K, Muntner P, Whelton PK, et al. (2005) Global burden of hypertension: analysis of worldwide data. Lancet 365: $217-$ 223.

23. Oommen AM, Abraham VJ, George K, Jose VJ (2016) Prevalence of risk 
Citation: Yazie D, Shibeshi W, Alebachew M, Berha AB (2018) Assessment of Blood Pressure Control among Hypertensive Patients in Zewditu Memorial Hospital, Addis Ababa, Ethiopia: A Cross-Sectional Study. J Bioanal Biomed 10: 80-87. doi:10.4172/1948-593X.1000210

factors for non-communicable diseases in rural \& urban Tamil Nadu. Indian J Med Res 144: 460-471.

24. Kiflie Y, Jira C, Nigussie D (2011) The quality of care provided to patients with chronic non-communicable diseases: A retrospective multi-setup study in Jimma Zone, southwest Ethiopia. Ethiop J Health Sci 21: 119-130.

25. Maziak W, Keil U, Doring A, Hense H (2003) Determinants of poor hypertension management in the community. J Hum Hypertens 17: 215-215.

26. Bakare O, Akinyinka M, Goodman O, Kuyinu Y, Wright O, et al. (2016) Antihypertensive use, prescription patterns, and cost of medications in a Teaching Hospital in Lagos, Nigeria. Nigerian journal of clinical practice 19: 668-672.

27. Gimpel NE, Schoj V, Rubinstein A (2006) Quality management of hypertension in primary care: do physicians treat patients' blood pressure level or cardiovascular risk?. Qual Prim Care 14: 211-217.

28. Ramli A, Miskan M, Ng K, Ambigga D, Nafiza M, et al. (2010) Prescribing of antihypertensive agents in public primary care clinics-Is it in accordance with current evidence? Malaysian family physician: Malays Fam Physician 5: 36-40.

29. Amare F (2016) Blood Pressure control and Associated Factors among Hypertensive Patients Attending Health Centers of Addis Ababa. Addis Ababa University.

30. Maghrabi IA (2013) Evaluation of antihypertensive prescribing patterns in the western region of Saudi Arabia and its compliance with national guidelines. Saudi J Health Sci 2: 118-126.

31. Filippi A, Paolini I, Innocenti F, Mazzaglia G, Battaggia A, et al. (2009) Blood pressure control and drug therapy in patients with diagnosed hypertension: a survey in Italian general practice. J Human Hypertens 23: 758-763.

32. Potchoo Y, Goe-Akue E, Damorou F, Massoka B, Redah D, et al. (2012) Effect of Antihypertensive Drug Therapy on the Blood Pressure Control among Hypertensive Patients Attending Campus' Teaching Hospital of Lome, Togo, West Africa. Pharmacol Pharma 3: 214-223.

33. Mulatu HA, Woldemichael M (2006) Pattern of Antihypertensive Therapy among Diabetic-Hypertensive Patients in Zewditu Memorial Hospital, Addis Ababa. Ethiop Med J 54: 77-82.

34. Abdulameer SA, Sahib MN, Aziz NA, Hassan Y, AIRazzaq HAA, et al. (2012) Physician adherence to hypertension treatment guidelines and drug acquisition costs of antihypertensive drugs at the cardiac clinic: a pilot study. Patien preference adherence 6: 101-108.

35. Gupta SK, Nayak RP, Rahavi R, Kumar A (2015) A cross-sectional retrospective study to assess the pattern of prescribing for inpatient hypertensive cases in a tertiary hospital and to find out the possible avenues for betterment of hypertension management. AMHS 3: 60-65.

36. Woldu MA, Shiferaw DF, Lenjisa JL, Tegegne GT, Tesafye G, et al. (2014) Antihypertensive medications pattern and their effect in blood pressure control in patients attending Bishoftu general hospital ambulatory ward, Debrezeit (Bishoftu), Ethiopia: World J Pharm Sci 2: 1198-1205.

37. Hasan MJ (2016) Pattern of drugs prescribed for treatment of hypertensive patients: Bangladesh. Afr J Pharm Pharmacol 10: 521-525.

38. Murti K, Khan MA, Dey A, Sethi MK, Das P, et al. (2015) Prescription Pattern of Anti-Hypertensive Drugs in Adherence to JNC-7 Guidelines. Am J Pharmacol Toxicol 10: 27-31.

39. Romday R, Gupta AK, Bhambani P (2016) An assessment of antihypertensive drug prescription patterns and adherence to joint national committee- 8 hypertension treatment guidelines among hypertensive patients attending a tertiary care teaching hospital. Int J Res Med Sci 4: 5125-5133.

40. Arief M, Harika B, Satyanarayana B, Pasha SW, Paladugu ND (2013) Evaluation of prescribing pattern of antihypertensive drugs in a tertiary care hospital. Acta Chim. Pharm. Indica 3: 172-181.

41. Adugna B (2015) Assessment of hypertension control and factors associated with the control among hypertensive patients attending at Zewditu Memorial Hospital, Ethiopia. Addis Ababa University.

42. Rachana $\mathrm{P}$, Anuradha $\mathrm{H}, \mathrm{MC}$ Shivamurthy (2014) Anti hypertensive prescribing patterns and cost analysis for primary hypertension: a retrospective study. J Clin Diagn Res 8: HC19-HC22.

43. The Food, Medicine and Health Care Administration and Control Authority (FMHACA) of Ethiopia (2014) Standard Treatment Guidelines For General Hospital 3rd edn

44. Odili V, Oghagbon E, Ugwa N, Ochei U, Aghomo O (2008) Adherence to international guidelines in the management of hypertension in a tertiary hospital in Nigeria. Trop J Pharm Res 7: 945-952.

45. Paulose R, Bairy K, Prabhu MM, Nayak V, Tripathy A (2017) Comparison of Efficacy and Adverse Drug Reactions of Monotherapy Versus Combination Therapy of Antihypertensives Among Diabetic Hypertensive Patients In A Tertiary Care Hospital. Asian J Pharm Clin Res 10: 385-391.

46. Kalamdani AR, Bhandare B, Hemamalini MB, Krishna MV (2014) A Prospective Study of Prescribing Pattern of Antihypertensive Drugs in Tertiary Care Hospital, Bangalore. J Evolution Med Dent Sci 2: 10339-10344.

47. Bakris GL, Williams M, Dworkin L, Elliott WJ, Epstein, M et al. (2000) Preserving renal function in adults with hypertension and diabetes: a consensus approach. National Kidney Foundation Hypertension and Diabetes Executive Committees Working Group. Am J Kidney Dis. 36: 646-661. 